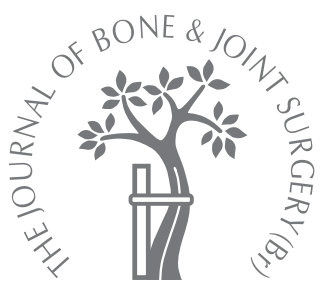

N. W. Emms, I. Stockley, A. J. Hamer, J. M. Wilkinson

From the Lower Limb Arthroplasty Unit, Department of Orthopaedics, Northern General Hospital, Sheffield, United Kingdom

\title{
Long-term outcome of a cementless, hemispherical, press-fit acetabular component
}

\author{
SURVIVORSHIP ANALYSIS AND DOSE-RESPONSE RELATIONSHIP \\ TO LINEAR POLYETHYLENE WEAR
}

\begin{abstract}
Between 1988 and 1998 we implanted 318 total hip replacements (THRs) in 287 patients using the Plasmacup (B. Braun Ltd, Sheffield, United Kingdom) and a conventional metalon-polyethylene articulation. The main indications for THR were primary or secondary osteoarthritis.
\end{abstract}

At follow-up after a mean $\mathbf{1 1 . 6}$ years ( 7.6 to 18.4) 17 patients had died and 20 could not be traced leaving a final series of 280 THRs in 250 patients. There were 62 revisions $(22.1 \%)$ in 59 patients. A total of 43 acetabular shells (15.4\%) had been revised and $13(4.6 \%)$ had undergone exchange of the liner. The most frequent indications for revision were osteolysis and aseptic loosening, followed by polyethylene wear. The mean Kaplan-Meier survival of the Plasmacup was $\mathbf{9 1 \%}$ at ten years and $\mathbf{5 8 \%}$ at 14 years. Osteolysis was found around $36(17.1 \%)$ of the 211 surviving shells. The median annual rate of linear wear in the surviving shells was $0.12 \mathrm{~mm} /$ year and $0.25 \mathrm{~mm} /$ year in those which had been revised $(p<0.001)$. Polyethylene wear was a strong independent risk factor for osteolysis and aseptic loosening. The percentage of patients with osteolysis increased proportionately with each quintile of wear-rate.

There is a high late rate of failure of the Plasmacup. Patients with the combination of this prosthesis and bearing should be closely monitored after ten years.

Cementless modular acetabular prostheses were introduced in the 1980 s as a response to the perceived poor results of cemented, allpolyethylene, acetabular implants. The intention was to eliminate cemented fixation, which was thought at the time to be the principal cause of osteolysis, and to make the transfer of load from the bearing surface to the pelvis more even. ${ }^{1,2}$ Since then it has become apparent that polyethylene wear, rather than 'cement disease', is the major cause of pelvic osteolysis. McCombe and Williams ${ }^{3}$ have shown in a randomised, clinical trial that a conventional polyethylene liner mounted in a metal shell has approximately twice the rate of annual linear wear of a cemented all-polyethylene component and would therefore be expected to have a higher rate of osteolysis. ${ }^{4}$

The Plasmacup (B Braun Ltd, Sheffield, United Kingdom) consists of a cementless, hemispherical, modular, titanium acetabular shell and a press-fit ultra-high-molecularweight polyethylene (UHMWPE) liner. Its outer surface has a porous plasma-sprayed titanium coating $0.35 \mathrm{~mm}$ thick. Primary stability is achieved by interference fit, and secondary stability by ongrowth of bone. Between
1988 and 2002 this prosthesis was used at our hospital for younger patients, generally under 60 years of age, undergoing primary total hip replacement (THR). Throughout this period the polyethylene liners were made of conventional UHMWPE and sterilised by gamma irradiation in an inert gas. In 2002, our short- to medium-term studies on the use of this prosthesis showed good results, with a survival of $97.7 \%$ at five years in 128 hips and a prevalence of radiological osteolysis of $3.0 \% .^{5}$ However, over the last five years we have seen an increasing incidence of acetabular osteolysis and high rates of annual linear wear (Fig. 1). We therefore undertook a review of the surviving patients who had undergone THR using this prosthesis between 1988 and 1997 in order to establish its long-term survivorship.

\section{Patients and Methods}

Between 1988 and 1997, 318 primary THRs were performed using the Plasmacup in 287 patients by a variety of surgeons of both consultant and training grade. In 2007, patients with a minimum follow-up of ten years were invited to attend for clinical and radiological review. By this stage 20 patients 


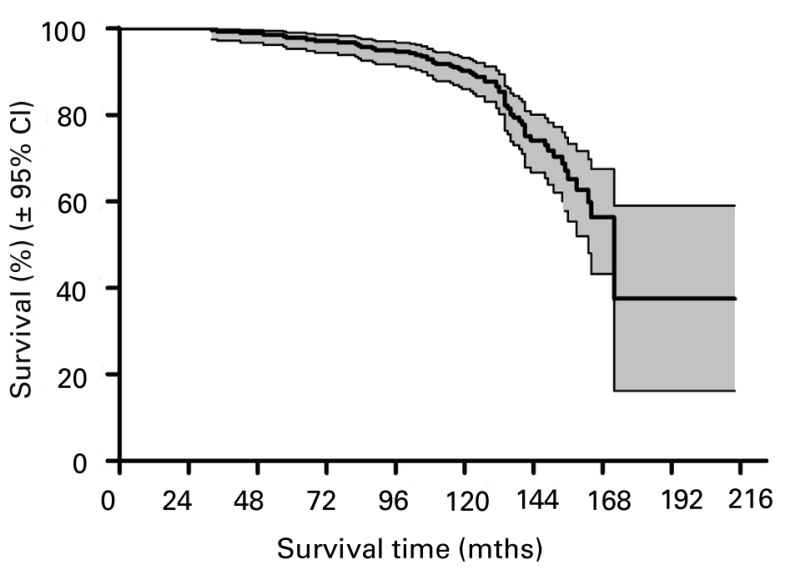

Fig. 1a

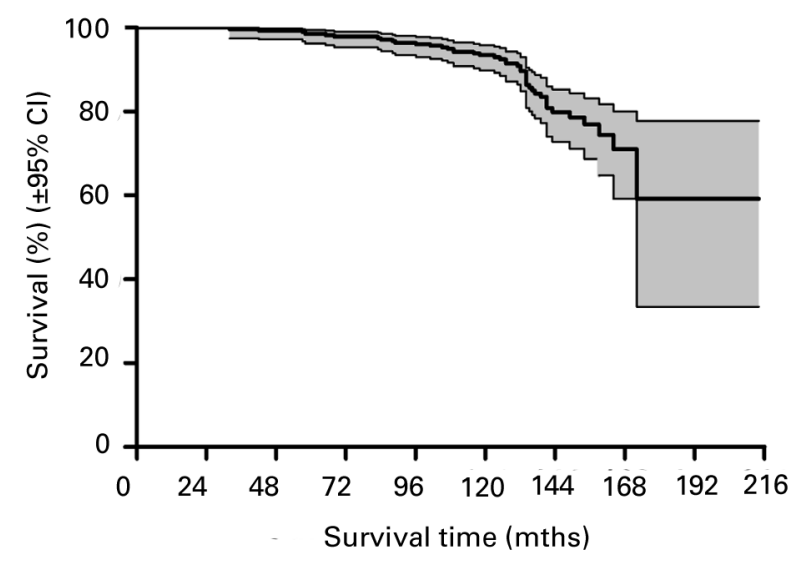

Fig. 1c

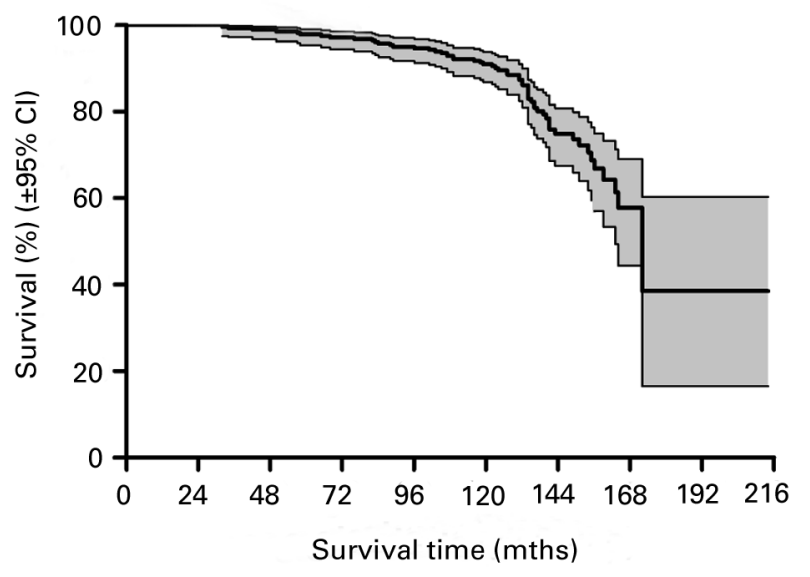

Fig. $1 b$

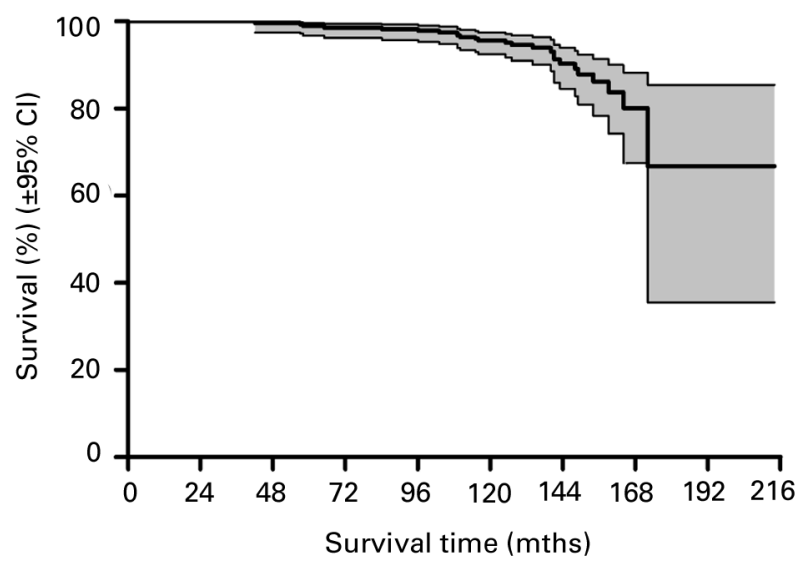

Fig. 1d

Kaplan-Meier survival analyses for the acetabular and femoral prostheses showing a) the overall revision-free survival for total hip replacement, b) revision-free survival of the Plasmacup shell and/or liner, c) revision-free survival of the Plasmacup shell and d) revision-free survival of the femoral prosthesis ( $95 \% \mathrm{Cl}, 95 \%$ confidence interval).

(21 hips) had been lost to follow-up and 17 (17 hips) had died from unrelated causes, leaving a final series of 280 hips in 250 patients. There were 125 men and 125 women in the group of whom 219 (245 hips) underwent clinical and radiological review. The remaining 31 (35 hips) were unable to attend but agreed to a telephone interview. This review was approved by the Audit Department of the Sheffield Teaching Hospitals NHS Foundation Trust.

The median age of the patients at surgery was 54 years (Interquartile range (IQR) 46 to 59, minimum 19 to maximum 77). The indication for THR was primary osteoarthritis in 204 hips, developmental dysplasia in 32, fracture of the hip in 16, inflammatory arthritis in seven, previous Perthes' disease in seven, avascular necrosis in six, as a conversion from an arthrodesis in four, previous slipped capital femoral epiphysis in three, and a neuromuscular disorder in one. All the procedures were performed in a laminar-flow theatre with the patient in the lateral decubitus position. The surgical approach was anterolateral in $243(86.8 \%)$ hips, posterior in $28(10.0 \%)$, and lateral with a trochanteric osteotomy in nine $(3.2 \%)$. The technique for insertion of the shell included under-reaming by $2 \mathrm{~mm}$ and pulsed lavage of the reamed acetabulum. The median diameter of the shell was $52 \mathrm{~mm}$ (interquartile range (IQR) 48 to 54). The median thickness of the liner was $13 \mathrm{~mm}$ (minimum 8; maximum 20; IQR 12 to 15). The femoral components used were the Exeter (Stryker Ltd, Staines, United Kingdom) in 194 hips, the Charnley (DePuy Ltd, Leeds, United Kingdom) in 53, the BiContact (B Braun Ltd) in 14, the TPS (DePuy Ltd) in 12, the Stanmore (Biomet, Swindon, United Kingdom) in five, Huckstep (B Braun Ltd) in one and Kent (Biomet) in one. All the cemented femoral components were inserted after pulsed lavage of the prepared femur with saline, and using vacuum-mixed Palacos $R$ cement containing gentamicin (Schering Plough Ltd, Welwyn Garden City, United Kingdom). This was introduced retrograde using a cement gun over a cement restrictor, and pressurised before insertion of the component. Cementless femoral component were inserted according to the manufacturers' instructions. A conventional UHMWPE acetabu- 
lar liner, gamma irradiated in an inert gas, was used in each hip. A $22 \mathrm{~mm}$ metal head was used in 142 hips and a 28 $\mathrm{mm}$ metal head in 138 .

At clinical review a standardised anteroposterior radiograph of the pelvis was taken with the beam centred on the symphysis pubis and with a focus-to-film distance of $100 \mathrm{~cm}$. A lateral radiograph was also taken. Linear wear of the liner was measured by a uni-radiological technique on digitised radiographs using EBRA digital software (EBRA-Cup version 2003; University of Innsbruck, Austria) according to a previously described protocol. ${ }^{6}$ In patients who had had their acetabular component revised, polyethylene wear was measured from the pre-revision radiograph by the same method. Loosening of the acetabular component was defined radiologically according to the criteria of Hodgkinson, Shelley and Wroblewski. ${ }^{7}$ Linear and expansile osteolytic lesions were recorded in the three zones described by DeLee and Charnley. ${ }^{8}$ Loosening of the femoral component was defined according to the criteria of Harris and McGann. ${ }^{9}$ Femoral osteolysis and radiolucent lines were recorded in the zones described by Gruen, McNeice and Amstutz. ${ }^{10}$

Statistical analysis. All analyses were two-tailed using SPSS statistical software version 15 (SPSS Inc., Chicago, Illinois). A p-value $\leq 0.05$ was deemed to be significant. The KaplanMeier survivorship analyses were calculated using Graphpad Prism version 5 (Graphpad Software Inc, La Jolla, California). Revision was used as the endpoint and was defined as "an operation that involved the removal and/or replacement of one or more components of a joint replacement". Continuous data were analysed using the MannWhitney $U$ test and categorical data were analysed using the chi-squared test. Acetabular revision was further subdivided into 'major' involving removal and/or replacement of the acetabular shell and 'minor' which did not involve the removal and/or replacement of the shell, such as an exchange of liner. Risk factors for revision of the Plasmacup were analysed using the Cox proportional hazards model.

\section{Results}

Survivorship of the prosthesis. The revision-free survival for any indication was $90.2 \%$ (95\% confidence interval (CI) 86.1 to 93.2 ) at ten years, but only $56.3 \%$ (95\% CI 43.2 to 67.6) at 14 years after operation (Fig. 1). The ten-year survival of the Plasmacup was $91.0 \%$ (95\% CI 86.9 to 93.8 ), but fell rapidly thereafter to $57.8 \%$ (95\% CI 44.4 to 69.0$)$ at 14 years. When minor revisions (liner exchange) were excluded, the survival of the Plasmacup shell at ten and 14 years was $93.5 \%$ (95\% CI 89.8 to 95.8 ) and $71.1 \%$ (95\% CI 59.2 to 80.0 ), respectively. The survival of the femoral component was $95.6 \%$ (95\% CI 92.4 to 97.5 ) and $80.2 \%(95 \%$ CI 72.1 to 92.8$)$ at ten and 14 years, respectively.

We have revised 62 hips $(22.1 \%)$ in 59 patients $(23.6 \%)$. Of these, $43(15.4 \%)$ required revision of the acetabular shell. In 24 of these 43 hips $(55.8 \%)$ only the shell was revised, in $18(41.8 \%)$ the femoral component and acetab- ular shell were revised, and in one hip $(2.3 \%)$ a liner exchange was followed by an exchange of both the shell and the femoral component. In 13 hips $(21.0 \%)$ only the liner was revised. Three hips had revision of both the liner and the femoral component, and in three only the femoral component was revised.

The indication for revision of the acetabular shell was osteolysis in 28 hips, infection in five, aseptic loosening in four, excessive wear with shell damage but without radiological osteolysis in four, and recurrent dislocation in two. The indication for liner exchange was wear in 13 hips and dislocation in four. The indication for revision of the femoral component was osteolysis in ten hips, aseptic loosening in five, infection in five, fracture of the component secondary to osteolysis in three, and wear damage of the femoral head in a well-fixed monobloc component in two.

Radiological complications in the surviving prostheses. In the 245 hips which had undergone radiological review, there were 211 surviving primary acetabular shells and 241 femoral components. Osteolysis was present around $36(17.1 \%)$ of the surviving shells. This was expansile in 28 , involving a single DeLee and Charnley zone in 17 hips and multiple zones in 11, and in eight the pattern of osteolysis was linear involving a single DeLee and Charnley zone. Osteolysis was present around $37(15.0 \%)$ of the surviving femoral components. In 14 hips this was expansile, involving a single Gruen zone in four hips and multiple zones in ten, and in 23 the pattern of osteolysis was linear involving a single Gruen zone in 12 and multiple zones in 11. One further femoral component was loose and migrated.

Polyethylene wear and other risk factors for revision. It was possible to measure the rate of polyethylene wear in 237 $(84.6 \%)$ hips. In the remainder, radiographs suitable for EBRA analysis were unavailable. The median rate of annual linear wear in those revised for any indication was $0.25 \mathrm{~mm} /$ year (IQR 0.18 to 0.32 ) compared with $0.12 \mathrm{~mm} /$ year (IQR 0.08 to 0.18 ) in those which remained in situ (Mann-Whitney $\mathrm{U}$ test, $\mathrm{p}<0.001)$. The median rate of annual linear wear in surviving Plasmacups with osteolysis was $0.16 \mathrm{~mm} /$ year (IQR 0.11 to 0.23 ) compared with $0.12 \mathrm{~mm} /$ year (IQR 0.07 to 0.17 ) for those without signs of osteolysis (Mann-Whitney $\mathrm{U}$ test, $\mathrm{p}=0.009)$. The median rate of wear in patients who had a $22 \mathrm{~mm}$ diameter femoral head was $0.15 \mathrm{~mm} /$ year (IQR 0.08 to 0.20 ) and $0.15 \mathrm{~mm} /$ year (IQR 0.09 to 0.22 ) in those with a head of $28 \mathrm{~mm}$ diameter $(\mathrm{p}=0.477)$.

The rate of polyethylene wear was an independent risk factor for revision of the acetabular shell (Cox proportional hazards model, $\mathrm{B}=3.89$, SEM 0.77, $\mathrm{p}<0.001)$. The age at surgery, gender, diagnosis, surgical approach, diameter of the shell, thickness of the liner, size and material of the femoral head ( $22 \mathrm{~mm} v s 28 \mathrm{~mm}$; stainless steel/cobalt-chrome), and the design of the femoral component were not risk factors for survival of the acetabular shell (Cox univariate analyses, all comparisons $\mathrm{p}>0.05$ ). These covariates were also not significant predictors of the rate of polyethylene wear (multiple linear regression, all comparisons $\mathrm{p}>0.05$ ). 


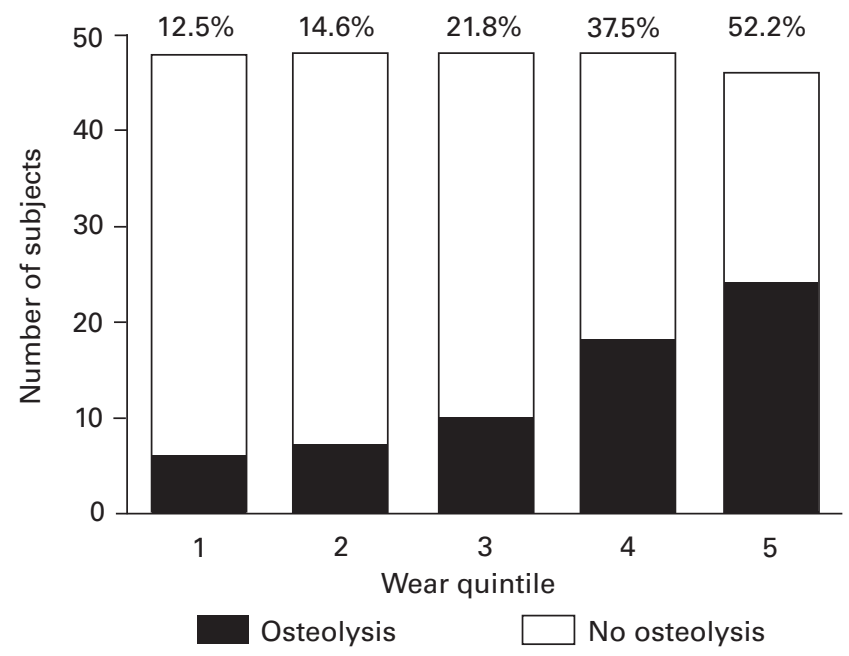

Fig. 2a

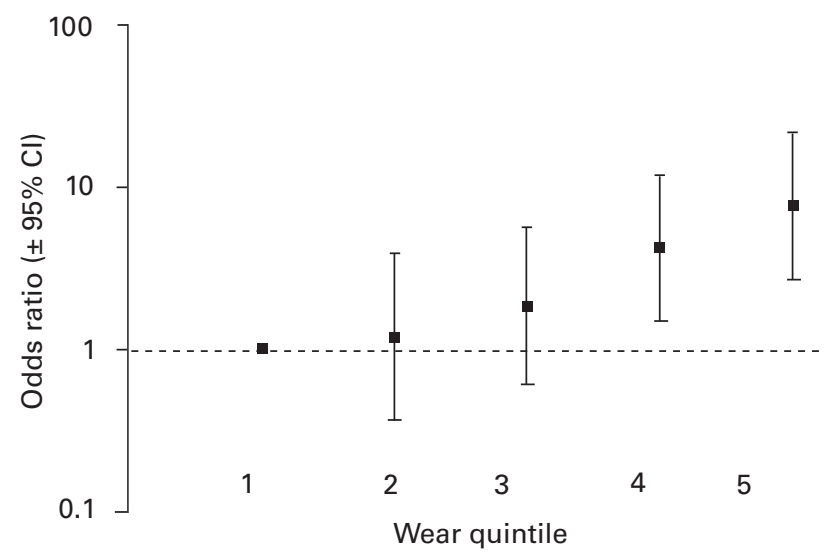

Fig. 2b

Figure 2a) a histogram showing the number of patients with osteolysis by quintile of the annual linear wear ( $\mathrm{n}=48$ subjects for quintiles 1 to 4 and $\mathrm{n}=46$ subjects for quintile 5 ). Figure $2 \mathrm{~b}$ ) graph showing the odds ratio $\pm 95 \%$ confidence interval (Cl) for osteolysis by quintile group versus quintile

1.

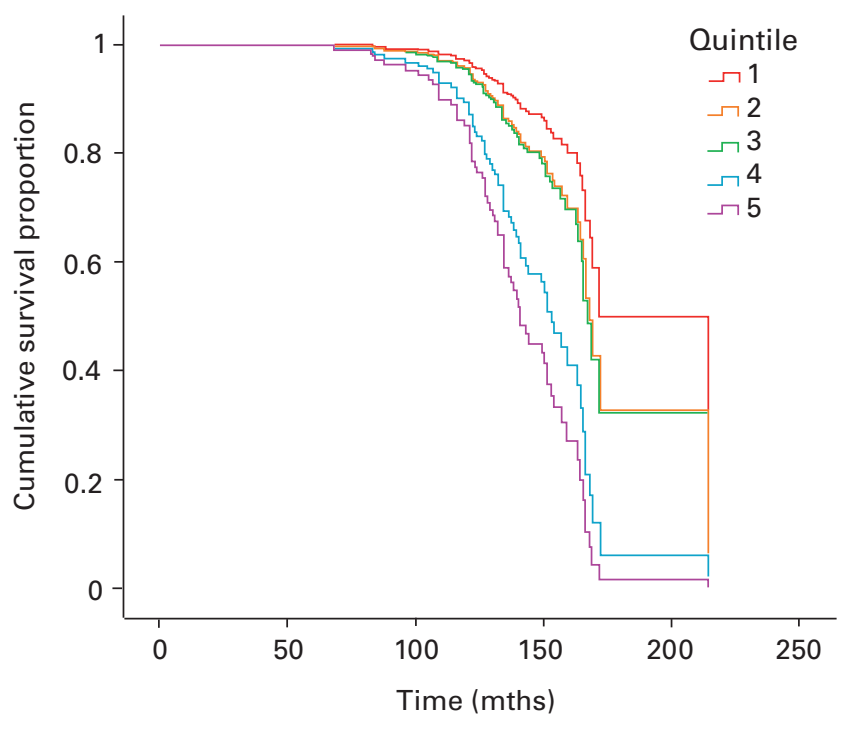

Fig. 3

Survivorship analysis made using the Cox proportional hazards model showing the effect of the wear rate quintile on osteolysis or survival free of aseptic loosening as defined by radiological diagnosis or revision after adjustment for the potential confounding effects of age at surgery, diagnosis, gender, diameter and material of the femoral head and thickness of the polyethylene liner.

The body mass index (BMI) and level of activity, as measured by the Oxford hip score, ${ }^{11}$ were assessed for patients who attended for clinical review with a surviving prosthesis. The mean BMI in this group was 27.8 (95\% CI 27.0 to 28.7) and the mean Oxford hip score 22.1 (20.4 to 23.9). Neither of these variables was a significant predictor of polyethylene wear in this group of survivors (linear regression $\mathrm{p}>0.05$ ).
The patients were divided into quintiles (q) based on the rate of annual linear wear, and the odds ratio was calculated using a method described previously. ${ }^{4}$ The lower and upper boundaries for each quintile (units $=\mathrm{mm} / \mathrm{yr}$ ) were 0.01 to 0.08 (q1), 0.08 to 0.12 (q2), 0.12 to 0.17 (q3), 0.17 to $0.24(\mathrm{q} 4)$, and 0.25 to 0.74 (q5). The proportion of patients who had revision for osteolysis, loosening or radiological evidence of osteolysis increased with each quintile from $12.5 \%$ for quintile 1 to $52.2 \%$ for quintile 5 (chisquared test, $\mathrm{p}<0.001$; Fig. 2a). The odds of osteolysis, loosening, or radiological evidence of osteolysis also increased with each quintile (Fig. 2b). Modelling of the effect of each quintile was also examined using survivorship analysis by the Cox proportional hazards model. This also showed a decline in osteolysis-free survival with increasing quintile (Fig. 3). This analysis was performed including age at surgery, gender, diagnosis, material and diameter of the femoral head, and thickness of the polyethylene liner as analysis covariates, and the resultant survivorship curves for each quintile are presented after adjustment for any potential confounding effects of these other variables.

\section{Discussion}

We previously reported a favourable early outcome with this prosthesis in 128 THRs from the current series. ${ }^{12}$ The survival rate was $98 \%$ at 59 months, with few radiological signs of osteolysis. These results were similar to those of Badhe, Quinnell and Howard, ${ }^{13}$ who showed a survival of the Plasmacup of $97 \%$ at 82 months in 153 hips. These findings were also similar to the mid-term results of studies on many other cementless, modular, acetabular shells using conventional polyethylene liners, including the Duraloc $300,{ }^{14}$ the Anatomic, ${ }^{15}$ and the Harris-Galante I and II. ${ }^{16,17}$ However, the survival of this type of bearing couple rapidly 
declined in our series after ten years. This was consistent with the findings of the Scandinavian joint registries, and suggested that the reporting of 'mid-term' survivorship is a poor guide to the long-term survival of this type of bearing couple. For example, according to the 2007 report of the Swedish hip register ${ }^{18}$ the ten-year survival of components implants in patients between the ages of 50 and 59 years at surgery was $90 \%$, but by 16 years had fallen to $78 \%$ in men and $68 \%$ in women. Similarly, the Finnish hip register showed that the ten-year survival of this type of component and bearing in patients aged between 55 and 64 years at surgery was $95 \%(95 \%$ CI 94.0 to 96.0$)$ at ten years, but $81 \%(95 \%$ CI 77.0 to 85.0$)$ at 15 years. ${ }^{19}$

Osteolysis and aseptic loosening are thought to result from an inflammatory osteolytic response to wear debris from the implants. The rate of wear and osteolysis are closely associated. ${ }^{20}$ The odds of revision or osteolysis in our series both increased with the rate of polyethylene wear in a 'dosedependent' manner. This relationship was seen both when using a simple analysis of the odds of osteolysis by wear-rate quintile 4 and when using survivorship analysis. This doseresponse relationship is consistent with our previous observations of the relationship between wear and osteolysis in cemented polyethylene acetabular components, ${ }^{4}$ and is further evidence that the idea of a 'wear-threshold' for osteolysis is invalid. ${ }^{21}$ Indeed, $27 \%$ of the patients with osteolysis in our series had a wear rate of $0.12 \mathrm{~mm} /$ year or less. Furthermore, this dose-response relationship resisted the potential confounding effects of age at surgery, gender, diagnosis, the size and material of the femoral head and the thickness of the polyethylene liner which were used as analysis covariates in the Cox regression model.

The wear rates which we observed in our series were similar to those previously reported for conventional polyethylene liners articulating with a metal head. McCombe and Williams, ${ }^{3}$ in a randomised trial of 162 patients, found a mean rate of wear over six years of $0.15 \mathrm{~mm} /$ year. Previous studies which have reported the annual wear of modular polyethylene liners have noted rates of from 0.11 to $0.32 \mathrm{~mm} /$ year. $^{14,22-27}$ These are approximately twice as great as those reported in cemented monobloc polyethylene acetabular components articulating against metal heads, which have wear rates in the range 0.05 to $0.11 \mathrm{~mm} /$ year. $^{3,20,28}$

Although osteolysis and revision of the acetabular shell were strongly associated with the rate of wear of the bearing surface, we found no association with other factors such as age at surgery, gender, indication for primary surgery, surgical approach, diameter of the shell, thickness of the liner, size and material of the femoral head or the design of the femoral component. Our finding that the size of the femoral head $(22 \mathrm{~mm} / 28 \mathrm{~mm})$ had no effect on the rate of osteolysis contradicts that of Livermore et al, ${ }^{28}$ but agrees with that of Hallan, Lie and Havelin. ${ }^{29}$ The likely reason for this lack of association is that, in our series, only $1 \%$ of liners were $\leq 8 \mathrm{~mm}$ thick and the median thickness was $13 \mathrm{~mm}$. Thus, our data did not suffer from the potential confounding effect of the use of liners of inadequate thickness in which the bulk mechanical properties of the material rather than the surface wear rate become the limiting factors.

Our data support the findings of others that conventional polyethylene liners in cementless shells have a high rate of wear and poor long-term survivorship. They also confirm a dose-response relationship between polyethylene wear and osteolysis which does not support the concept of a 'safe' threshold for the wear rate. Furthermore, the rapid decline in the survivorship of the prosthesis after ten years suggests limitations in the ten-year benchmarking classification system used by the National Institute of Clinical Excellence (NICE) ${ }^{30}$ in the United Kingdom.

No benefits in any form have been received or wil be received from a commercial party related directly or indirectly to the subject of this article.

\section{References}

1. Goldring SR, Schiller AL, Roelke $\mathbf{M}$, et al. The synovial-like membrane at the bone-cement interface in loose total hip replacements and its proposed role in bone lysis. J Bone Joint Surg [Am] 1983;65-A:575-84.

2. Carter DR, Vasu R, Harris WH. Stress distributions in the acetabular region. II: effects of cement thickness and metal backing of the total hip acetabular component. J Biomech 1982;15:165-70.

3. McCombe $\mathbf{P}$, Williams SA. A comparison of polyethylene wear rates between cemented and cementless cups: a prospective, randomised trial. J Bone Joint Surg [Br] 2004;86-B:344-9.

4. Wilkinson JM, Hamer AJ, Stockley I, Eastell R. Polyethylene wear rate and osteolysis: critical threshold versus continuous dose-response relationship. J Orthop Res 2005;23:520-5

5. Gordon A, Wilkinson JM, Stockley I. Midterm outcome of the plasma cup in total hip arthroplasty. Hip Internationa/ 2002;12:119-25.

6. Wilkinson JM, Hamer AJ, Elson RA, Stockley I, Eastell R. Precision of EBRADigital software for monitoring implant migration after total hip arthroplasty. $J$ Arthroplasty 2002;17:910-16

7. Hodgkinson JP, Shelley P, Wroblewski BM. The correlation between the roentgenographic appearance and operative findings at the bone-cement junction of the socket in Charnley low friction arthroplasties. Clin Orthop 1988;228:105-9.

8. DeLee JG, Charnley J. Radiological demarcation of cemented sockets in total hip replacement. Clin Orthop 1976;121:20-32.

9. Harris WH, McGann WA. Loosening of the femoral component after use of the medullary-plug cementing technique: follow-up note with a minimum five-year follow-up. J Bone Joint Surg [Am] 1986;68-A:1064-6.

10. Gruen TA, McNeice GM, Amstutz HC. "Modes of failure" of cemented stem-type femoral components: a radiographic analysis of loosening. Clin Orthop 1979;141:17-27.

11. Dawson J, Fitzpatrick R, Carr A, Murray D. Questionnaire on the perceptions of patients about total hip replacement. J Bone Joint Surg [Br] 1996;78-B:185-90.

12. Wilkinson JM, Gordon A, Stockley I. Experiences with the Plasmacup: early stability, wear, remodelling, and outcome. Int Orthop 2003;27(Suppl 1):16-19.

13. Badhe NP, Quinnell RC, Howard PW. The uncemented Bi-Contact total hip arthroplasty. J Arthroplasty 2002;17:896-901.

14. Grobler GP, Learmonth ID, Bernstein BP, Dower BJ. Ten-year results of a pressfit, porous-coated acetabular component. J Bone Joint Surg [Br]2005;87-B:786-9.

15. Udomkiat P, Dorr LD, Wan Z. Cementless hemispheric porous-coated sockets implanted with press-fit technique without screws: average ten-year follow-up. $J$ Bone Joint Surg [Am] 2002;84-A:1195-200

16. Clohisy JC, Harris WH. The Harris-Galante porous-coated acetabular component with screw fixation: an average ten-year follow-up study. J Bone Joint Surg [Am] 1999;81-A:66-73

17. Archibeck MJ, Berger RA, Jacobs JJ, et al. Second-generation cementless total hip arthroplasty: eight to eleven-year results. J Bone Joint Surg [Am] 2001;83-A:1666-73.

18. No authors listed. Swedish Hip Register Annual Report 2007. http:// www.jru.orthop.gu.se (date last accessed 21 April 2010).

19. Mäkelä KT, Eskelinen A, Pulkkinen P, Paavolainen P, Remes V. Total hip arthroplasty for primary osteoarthritis in patients fifty-five years of age or older: an analysis of the Finnish arthroplasty registry. J Bone Joint Surg [Am] 2008;90A:2160-70. 
20. Sochart DH. Relationship of acetabular wear to osteolysis and loosening in total hip arthroplasty. Clin Orthop 1999;363:135-50.

21. Harris WH. "The lysis threshold": an erroneous and perhaps misleading concept? J Arthroplasty 2003;18:506-10.

22. Duffy P, Sher JL, Partington PF. Premature wear and osteolysis in an HAcoated, uncemented total hip arthroplasty. J Bone Joint Surg [Br]2004;86-B:34-8.

23. Kim YH. Long-term results of the cementless porous-coated anatomic total hip prosthesis. J Bone Joint Surg [Br] 2005;87-B:623-7.

24. Tompkins GS, Jacobs JJ, Kull LR, Rosenberg AG, Galante J0. Primary total hip arthroplasty with a porous-coated acetabular component: seven-to-ten-year results. $J$ Bone Joint Surg [Am]1997;79-A:169-76.

25. Crowther JD, Lachiewicz PF. Survival and polyethylene wear of porous-coated acetabular components in patients less than fifty years old: results at nine to fourteen years. J Bone Joint Surg [Am] 2002;84-A:729-35.
26. Shih CH, Lee PC, Chen JH, et al. Measurement of polyethylene wear in cementless total hip arthroplasty. J Bone Joint Surg [Br] 1997;79-B:361-5.

27. Goosen JH, Verheyen CC, Tulp NJ. Mid-term wear characteristics of an uncemented acetabular component. J Bone Joint Surg [Br] 2005;87-B:1475-9.

28. Livermore J, Ilstrup D, Morrey B. Effect of femoral head size on wear of the polyethylene acetabular component. J Bone Joint Surg [Am] 1990;72-A:518-28.

29. Hallan G, Lie SA, Havelin LI. High wear rates and extensive osteolysis in 3 types of uncemented total hip arthroplasty: a review of the PCA, the Harris Galante and the Profile/Tri-Lock Plus arthroplasties with a minimum of 12 years median follow-up in 96 hips. Acta Orthop 2006;77:575-84

30. No authors listed. Guidance on the Selection of Prostheses for Primary Total Hip Replacement (TA2). National Institute for Clinical Excellence: Technology appraisals; Issued April 2000. http://www.nice.org.uk (date last accessed 21 April 2010). 